\title{
WIENER-HOPF OPERATORS AND ABSOLUTELY CONTINUOUS SPECTRA. II
}

\author{
BY C. R. PUTNAM ${ }^{1}$
}

Communicated by Maurice Heins, November 1, 1967

1. This paper is a continuation of [4]. It may be recalled that if $A$ is a self-adjoint operator on a Hilbert space $\mathfrak{S}$ with spectral resolution $A=\int \lambda d E_{\lambda}$, then the set of elements $x$ in $\mathfrak{g}$ for which $\left\|E_{\lambda} x\right\|^{2}$ is an absolutely continuous function of $\lambda$ is a subspace, $\mathfrak{S}_{a}(A)$, of $\mathfrak{S}$ (see, e.g., Halmos [1, p. 104]). The operator $A$ is said to be absolutely continuous if $\mathfrak{W}_{a}(A)=\mathfrak{W}$. As in [4], both spaces $L^{2}(0, \infty)$ and $L^{2}(-\infty, \infty)$ will be considered, but the underlying Hilbert space for the integral operators $T$ and $A$ occurring below will be $\mathfrak{S}=L^{2}(0, \infty)$. As in [4], let $k(t)$ on $-\infty<t<\infty$ satisfy

$$
k \in L^{1}(-\infty, \infty) \cap L^{2}(-\infty, \infty) \text { and } k(-t)=\bar{k}(t),
$$

and let $K(\lambda)$ denote the (real-valued) function

$$
K(\lambda)=\int_{-\infty}^{\infty} k(t) e^{i \lambda t} d t, \quad-\infty<\lambda<\infty .
$$

If the (bounded) operator $T$ on $\mathfrak{S}$ is defined by

$$
(T f)(t)=\int_{0}^{t} k(s-t) f(s) d s, \quad 0 \leqq t<\infty,
$$

then the self-adjoint operator $A=T+T^{*}=2 \operatorname{Re}(T)$ is given by

$$
(A f)(t)=\int_{0}^{\infty} k(s-t) f(s) d s
$$

There will be proved the following

THEOREM. If $k(t)$ satisfies (1) and if $k(t) \not \equiv 0$ (a.e.) on $-\infty<t<\infty$, then the self-adjoint operator $A$ of (4) is absolutely continuous and its spectrum is the closed interval

$$
\operatorname{sp}(A)=[\inf K(\lambda), \sup K(\lambda)],
$$

where $K(\lambda)$ is defined in (2).

In [4] the absolute continuity of $A$ was established under the hypothesis that $K(\lambda) \neq 0$ a.e. According to the above Theorem how-

\footnotetext{
1 This work was supported by a National Science Foundation research grant.
} 
ever, this property holds provided only that $K(\lambda) \not \equiv 0$, equivalently, that $k(t) \not \equiv 0$ a.e. In other words, $A$ is absolutely continuous except in the trivial case $A=0$. (Similar assertions hold for self-adjoint Toeplitz operators; see [3, pp. 132-133] for a discussion and references.) ${ }^{2}$ The relation (5) can be deduced from a theorem given in Krein [2, p. 224], concerning Wiener-Hopf operators on the halfline. However, this fact, as well as the assertions of the above Theorem concerning the absolute continuity, will be derived as consequences of general results on hyponormal operators on a Hilbert space and which are contained in the following

Lemma. Let $T$ be a bounded operator on a Hilbert space $\mathfrak{S}$ and let

$$
T^{*} T-T T^{*}=C, \quad C \geqq 0 .
$$

If $A=T+T^{*}$ then $\mathfrak{S}_{a}(A)$ contains the smallest subspace, $\mathfrak{M}_{T}$, of $\mathfrak{S}$ which reduces $T$ and which contains the range of $C$. Moreover, the spectrum of the real part of $T$ (that is, of $\frac{1}{2} A$ ) is the projection onto the real axis of the spectrum of $T$.

The proof of the Lemma can be found in [3, pp. 42-43, 46-47]. The proof of the absolute continuity assertion of the Theorem will be given in $\$ 2$ and that of $(5)$ in $\$ 3$.

2. It will be convenient to recall a part of the argument given in [4]. It was noted there that relation (6) holds for $T$ of (3) on $\mathfrak{S}=L^{2}(0, \infty)$ with $C=B^{*} B$ and $(B f)(t)=\int_{0}^{\infty} k(t+s) f(s) d s$. For $f \in L^{2}(0, \infty)$, put $\hat{f}(\lambda)=\int_{0}^{\infty} e^{-i \lambda t} f(t) d t \equiv F_{-}(\lambda)$ and $F_{+}(\lambda)=\int_{0}^{\infty} e^{i \lambda t} f(t) d t$ and let $R_{+}$and $R_{-}$denote the orthogonal subspaces of $L^{2}(-\infty, \infty)$ consisting of the elements $F_{+}$and $F_{-}$respectively. (Note that $R_{+}\left[R_{-}\right]$ can be regarded as the space of Fourier transforms of elements in $L^{2}(-\infty, \infty)$ which are 0 on the right [left] half-line.)

As in [4], if the space $\mathfrak{M}_{T}$ of the Lemma is not $\mathfrak{S}$, then there exists a function $q \in \mathfrak{S}, q \neq 0$ (that is, $q(t) \neq 0$ a.e. on $0 \leqq t<\infty$ ) such that $q \perp \mathfrak{M}_{T}$. (It will be shown below that necessarily $A=0$ in this case.) If $Q=Q(\lambda)=\int_{0}^{\infty} e^{-i \lambda t} q(t) d t\left(\in R_{-}\right)$, then, as was shown in [4], $Q \perp \bar{K}_{+}^{n} R_{+}$, $n=0,1,2, \cdots$, where

${ }^{2}$ Added in proof. It follows from a result of M. Rosenblum (Self-adjoint Toeplitz operators, 1965 Summer Institute in Spectral Theory and Statistical Mechanics, Brookhaven National Laboratory, Upton, New York) that the above $A$ is unitarily equivalent to a self-adjoint Toeplitz matrix. The absolute continuity of $A$ as well as the assertion (5) can then be deduced from the corresponding properties of Toeplitz operators; cf. [3] for a further discussion. This unitary equivalence is not used in the methods of the present paper however. 


$$
K_{+}(\lambda)=\int_{0}^{\infty} e^{i \lambda t} k(t) d t
$$

Since $Q \perp R_{+}$, it follows that

$$
Q \perp\left(\operatorname{Re}\left(K_{+}\right)\right)^{n} R_{+} \text {and } Q \perp\left(\operatorname{Im}\left(K_{+}\right)\right)^{n} R_{+} \text {for } n=0,1,2, \cdots
$$

Only the first relation of (9) was exploited in [4], where it was shown that, as a consequence, $Q(\lambda)=0$ a.e. on the set for which $\operatorname{Re}\left(K_{+}(\lambda)\right) \neq 0$. The same argument (involving Weierstrass' approximation theorem) shows however that $Q(\lambda)=0$ a.e. also on the set for which $\operatorname{Im}\left(K_{+}(\lambda)\right) \neq 0$. Since $q(t) \not \equiv 0$ a.e. on $0 \leqq t<\infty$, then $Q(\lambda) \not \equiv 0$ a.e. on $-\infty<\lambda<\infty$, and hence $K_{+}(\lambda)=0$ on a set of positive measure. However, since $k \in L^{1}(-\infty, \infty), K_{+}(\lambda)$ is the boundary function of a function $K_{+}(z)=\int_{0}^{\infty} e^{i z t} k(t) d t$ analytic in the upper half-plane $\operatorname{Im}(z)>0$ and bounded and continuous on $\operatorname{Im}(z) \geqq 0$. If the half-plane $\operatorname{Im}(z) \geqq 0$ is mapped onto the unit circle $|w| \leqq 1$ by the linear fractional transformation $w=(z-i) /(z+i)$, one then obtains a function $K_{+}(z(w))$ analytic in $|w|<1$, bounded on $|w| \leqq 1$, and continuous on $|w| \leqq 1$ except possibly at $w(\infty)=1$, and which is 0 on the boundary $|w|=1$ on a set of positive measure. It follows from the classical theorem of F. and M. Riesz [5] that this function must be identically 0 and hence, in particular, that $K_{+}(\lambda) \equiv 0$ on $-\infty<\lambda<\infty$. Hence $K(\lambda)=2 \operatorname{Re}\left(K_{+}(\lambda)\right) \equiv 0$ and so $k(t) \equiv 0$ a.e., that is, $A=0$. This completes the proof of the first portion of the Theorem.

3. To prove (5), let $z$ satisfy $0<|z|<1$ and put $\lambda=\int_{0}^{\infty} k(t) z^{t} d t$. (Here $z^{t}=e^{t \log z}$ where $\log z$ denotes any value of the logarithm function.) If $f(t)=z^{t}$, then $f \in \mathfrak{S}$ and one has

$$
\left(T^{*} f\right)(t)=\int_{t}^{\infty} k(s-t) z^{\bullet} d s=\int_{0}^{\infty} k(s) z^{t+\bullet} d s=\lambda f(t) .
$$

Thus the range of $\int_{0}^{\infty} k(t) z^{t} d t$, for $0<|z|<1$, belongs to the spectrum, even the point spectrum, of $T^{*}$. (This fact and its derivation (10) are analogous to corresponding results for Toeplitz matrices, with the integrals replaced by power series, due to Wintner [6]; cf. also [3, p. 129].) Since the spectrum is a closed set, it follows that $K_{+}(\lambda)$ of (8) is in the spectrum of $T^{*}$ for all real $\lambda$. Since $K(\lambda)=K_{+}(\lambda)+\bar{K}_{+}(\lambda)$, it follows from the second part of the Lemma that the interval [inf $K(\lambda)$, sup $K(\lambda)$ ] is certainly contained in the spectrum of $A=T$ $+T^{*}$. (Only this much of the Lemma will be needed here.) On the other hand, as was shown in [4], $(T f)^{\wedge}(\lambda)=\bar{K}_{+}(\lambda) \hat{f}(\lambda)$ and so, by the Parseval relation, 


$$
(A f, f)=(T f, f)+(\overline{T f, f})=\int_{-\infty}^{\infty} K(\lambda)|\hat{f}(\lambda)|^{2} d \lambda
$$

Hence, the spectrum of $A$ is clearly a subset of [inf $K(\lambda)$, sup $K(\lambda)$ ] and the relation (5) is proved.

\section{REFERENCES}

1. P. R. Halmos, Introduction to Hilbert space, Chelsea, New York, 1951.

2. M. G. Krein, Integral equations on a half-line with kernel depending upon the difference of the arguments, Amer. Math. Soc. Transl. 22 (1962), 163-288.

3. C. R. Putnam, Commutation properties of Hilbert space operators and related topics, Ergebnisse der Math. 36, Springer, Berlin, 1967.

4. Wiener-Hopf operators and absolutely continuous spectra, Bull. Amer. Math. Soc. 73 (1967), 659-662.

5. F. Riesz and M. Riesz, Über die Randwerte einer analytischen Funktion, Quat. Cong. des Math. Scand., Stockholm (1916), 27-44.

6. A. Wintner, Zur Theorie der beschränten Bilinearformen, Math. Z. 30 (1929), 228-282.

Purdue University 